\title{
A new fast track-fit algorithm based on broken lines
}

\author{
V. Blobel \\ Institut für Experimentalphysik, Universität Hamburg, Germany
}

\begin{abstract}
The determination of the charged particle momentum in HEP experiments requires a fit of a parametrization to the points measured in a tracking chamber. A new non-recursive track-fit algorithm based on broken lines allows to reconstruct the particle trajectory taking into account details of multiple scattering. It provides optimal parameters and their covariance matrices at track start and end, and optimal values at each measured point along the trajectory including the variances. The parametrization of the trajectory allows the use of sparse-matrix techniques with a total computing time proportional to the number of data points. Under test conditions the new algorithm is found to be six times faster than the Kalman filter.
\end{abstract}

Key words: Track fitting, Multiple scattering, Global least-squares fit

\section{Track measurement in particle physics}

For a HEP tracking detector in a typical collider experiment with a homogeneous magnetic field $\boldsymbol{B}_{z}$ (in $z$-direction) the ideal track parametrization is a helix with five parameters: the curvature $\kappa$ (inverse radius, signed), the distance $d_{c a}$ and the angle $\phi_{0}$ at the point of closest approach to the axis, the intercept $z_{0}$ and the slope parameter $\tan \lambda \equiv$ $\cot \vartheta$. Various effects can result in deviations to an ideal helix curve, and the track fit with a pure helix parametrization is not optimal. Multiple scattering deflections will influence all downstream measurement in a correlated way, and delimit the accuracy of momentum measurement at low momenta. In addition, there may be effects due to the inhomogeneity of the magnetic field and continuous en-

\footnotetext{
Email address: volker.blobel@desy.de (V. Blobel).

URL: www.desy.de/ blobel (V. Blobel).
}

ergy loss along the trajectory (radiation in case of electrons).

Different methods of track fitting [2] exist which are able to take the effects mentioned above into account. In global methods with a computing time $\propto$ $n^{3}$, where $n$ is the number of data points, the track parameters are determined in a single step. In the matrix method all effects of multiple scattering are included in the covariance matrix of the measured points, which becomes non-diagonal; in the breakpoint method a certain number of scattering planes is defined, increasing the number of parameters, while the covariance matrix of the measured points remains diagonal. In the progressive method [3] the track is followed by incorporating measurement after measurement with updating the parameter vector and covariance matrix, starting from an outer detector. The method is equivalent to the Kalman filter [4] and has become the standard method of track fitting; multiple scattering is introduced as 
process noise and optimal track parameters are determined at both ends of the track by smoothing in the direction opposite to the filter. These methods have a computing time $\propto n$ and hence are faster by a large factor compared to the global methods mentioned before.

The method proposed here can be considered as a global method too. First approximate track parameters are determined from simple $2 \mathrm{D}$ fits of a circle $([5])$

$$
\begin{aligned}
& \frac{1}{2} \kappa\left(x_{i}^{2}+y_{i}^{2}+d_{\text {ca }}^{2}\right) \\
& \quad-\left(1+\kappa d_{\text {ca }}\right)\left(x_{i} \sin \phi_{0}-y_{i} \cos \phi_{0}\right)+d_{\text {ca }}=0
\end{aligned}
$$

and of a straight line

$$
z_{i}=z_{0}+(\tan \lambda) \cdot s_{i}
$$

to the measured points. Then residuals w.r.t. the circle and the straight line are calculated as a function of the track length $s_{i}$ (in the $r \phi$-plane); a detailed fit to the residuals taking into account multiple scattering (and perhaps other effects) is made, where corrections to the track parameters like $\Delta \kappa$ are determined. Due to the special parametrization of the trajectory in the residual fit the computing time is $\propto n$ and the method is, under test conditions, faster by a factor of six, compared to the Kalman filter and smoothing. Results for the track parameters are almost identical for the global and Kalman methods. An example for a track fit is shown in Fig. 4. A circle fit is shown on the left, and the residuals to the circle are shown on the right as a function of the track length; the fit of the residuals is discussed in later sections.

\section{Multiple scattering}

A charged particle traversing material will make a large number of small angle collisions, called multiple scattering, which is dominated by Coulomb scattering off the nuclei. Multiple scattering is parametrized by two mutually orthogonal, uncorrelated angles. The Review of Particle Properties PDG [1] quotes the formula

$$
V[\theta]=\theta_{0}^{2}=\left(\frac{13.6 \mathrm{MeV}}{\beta p c}\right)^{2} t[1+0.038 \ln t]^{2}
$$

for the variance of the deflection angle $\theta$ of a singly charged particle with momentum $p$ and velocity $\beta$. The quantity $t$ is the thickness of the material in units of the radiation length $X_{0}$, thus $t=\Delta s / X_{0}$.

The trajectory of a charged particle traversing a homogeneous medium of thickness $\Delta s$ between two detector planes is shown in Fig. 1. The effect of multiple scattering after the traversal of a homogeneous medium of thickness $\Delta s$ can be described by two parameters, the deflection angle $\theta_{\text {plane }}$, or short $\theta$, and the angle $\psi$. This is the angle between the original particle direction and the straight line between the two intersection points (circles). The two angles are statistically correlated with a covariance matrix

$$
V\left[\begin{array}{l}
\theta \\
\psi
\end{array}\right]=\left(\begin{array}{cc}
1 & 1 / 2 \\
1 / 2 & 1 / 3
\end{array}\right) \theta_{0}^{2}
$$

In an ideal detector the intersections could be mea-

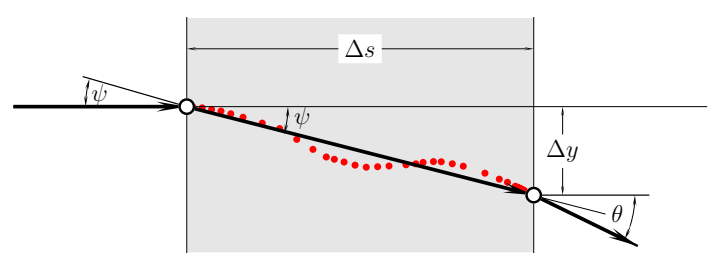

Fig. 1. Quantities used to describe multiple scattering.

sured with high precision and the straight line between the two intersections gives the complete information on the particle trajectory, which is available from the measurement. The angle between the direction of this line and the true particle direction is $\psi_{\text {left }} \equiv \psi$ on the left and $\psi_{\text {right }} \equiv \theta-\psi$ on the right of the medium. Expectation and variance of these two angles are identical with

$$
E\left[\begin{array}{c}
\psi_{\text {left }} \\
\psi_{\text {right }}
\end{array}\right]=\left(\begin{array}{l}
0 \\
0
\end{array}\right) \quad V\left[\begin{array}{c}
\psi_{\text {left }} \\
\psi_{\text {right }}
\end{array}\right]=\left(\begin{array}{cc}
1 / 3 & 1 / 6 \\
1 / 6 & 1 / 3
\end{array}\right) \theta_{0}^{2}
$$

for a homogeneous medium between the two detector planes. Usually the material distribution between two detector planes is inhomogeneous and the covariance matrix has to be calculated from the geometry of the material distribution within the $i$-th layer: 


$$
\boldsymbol{V}\left[\begin{array}{c}
\psi_{\text {left }} \\
\psi_{\text {right }}
\end{array}\right]_{i}=\left(\begin{array}{cc}
V_{L, i} & V_{L R, i} \\
V_{L R, i} & V_{R, i}
\end{array}\right),
$$

where the matrix elements are proportional to the value of $\theta_{0}^{2}$, calculated from $t$ using Eq. (1).

\section{Tracking in the $s z$-plane}

The approximate value of the momentum $p$ determined in simple $2 \mathrm{D}$ fits allows to calculate the multiple scattering variances $V[\theta]$. In the fit of the residuals in the $s z$-plane corrections to the parameters $z_{0}$ and $\tan \lambda$ are determined, taking multiple scattering into account.

Fig. 2 shows the trajectory of a charged particle with multiple scattering, and the intersection points of the trajectory with the detector planes. The coordinates $y_{i}$, with standard deviation $\sigma_{i}$, represent the residuals of the measurements at the detector planes with coordinates $s_{i}$, for $i=1,2, \ldots n$; they are transverse to the average track, and are uncorrelated. For an improved fit

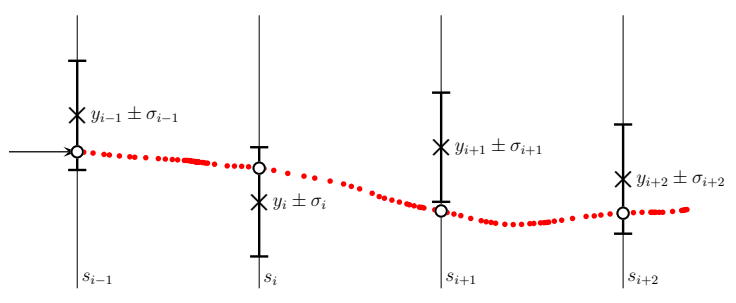

Fig. 2. Particle trajectory and measured residuals $y_{i}$.

taking into account the multiple scattering effects the new track-fit method developed here uses two phases in the track reconstruction:

Reconstruction of the trajectory: The trajectory, represented by the intersection points of the trajectory with the detector planes, is determined in a least squares fit; the estimates of the intersection points are denoted by $u_{i}$.

Track parameter determination: From the fitted $u_{i}$-values the two track parameters intercept and slope, required for the physics analysis, are determined at both sides of the track.

The least squares fit thus has $n$ parameters $u_{i}$ to be determined, one for each measured value $y_{i}$. The sum of squares to be minimized includes the sum of $\left(y_{i}-u_{i}\right)^{2} / \sigma_{i}^{2}$. The intersection points $u_{i}$ to be fitted are shown in Fig. 3. Each two adjacent points are

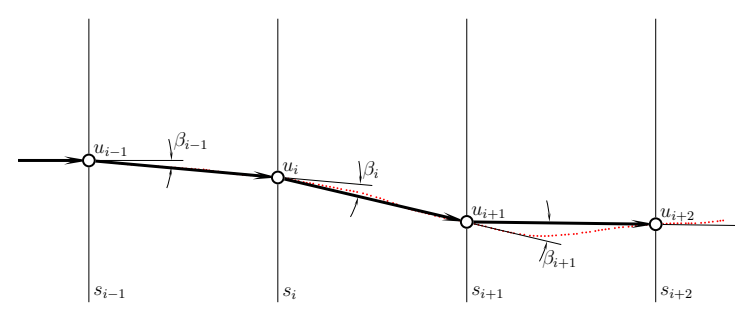

Fig. 3. Particle trajectory with fitted residuals $u_{i}$ and kink angles $\beta_{i}$.

connected by a straight line, and due to multiple scattering there is a kink angle,

$$
\beta_{i}=\psi_{\text {right }, i-1}-\psi_{\text {left }, i}
$$

between the straight line segments. Expectation values $E\left[\beta_{i}\right]$ are zero and the variances $V\left[\beta_{i}\right]$ are the sum of the variances of the angles $\psi_{\text {left }}$ and $\psi_{\text {right }}$ of the layers between detector planes (Eq. (3)):

$$
V\left[\beta_{i}\right]=\sigma_{\beta, i}^{2}=V\left[\psi_{\text {right }, i-1}\right]+V\left[\psi_{\text {left }, i}\right] .
$$

The approximation of the true trajectory is given by the points $\left(s_{i}, u_{i}\right)$. There are $(n-2)$ kink angles $\beta_{i}$, which are (to good approximation) linear functions of the values $u_{i-1}, u_{i}$ and $u_{i+1}$,

$$
\beta_{i}=\left[u_{i-1} \delta_{i-1}-u_{i}\left(\delta_{i-1}+\delta_{i}\right)+u_{i+1} \delta_{i}\right]
$$

with the definition $\delta_{i}=1 /\left(s_{i+1}-s_{i}\right)$. The $(n-2)$ angles $\beta_{i}$ all have an expectation value of zero and a variance given by the multiple scattering theory. This information is included in a least squares fit by minimizing the function

$$
S(\boldsymbol{u})=\sum_{i=1}^{n} \frac{\left(y_{i}-u_{i}\right)^{2}}{\sigma_{i}^{2}}+\sum_{i=2}^{n-1} \frac{\beta_{i}^{2}}{\sigma_{\beta, i}^{2}}
$$

with respect to the values $u_{i}$; no explicit parametrization of the trajectory is defined.

The vector $\boldsymbol{u}$ that minimizes the sum-expression $S(\boldsymbol{u})$ is given by the solution of the standard normal equations $\boldsymbol{C}_{u} \boldsymbol{u}=\boldsymbol{r}$ of linear least squares. The elements of matrix $\boldsymbol{C}_{u}$ and of vector $\boldsymbol{r}$ are given by sums, calculated in a loop over all measured data. The matrix $\boldsymbol{C}_{u}$ is a symmetric band matrix, where all elements outside a narrow band (bandwidth $m=2$ ) vanish: 

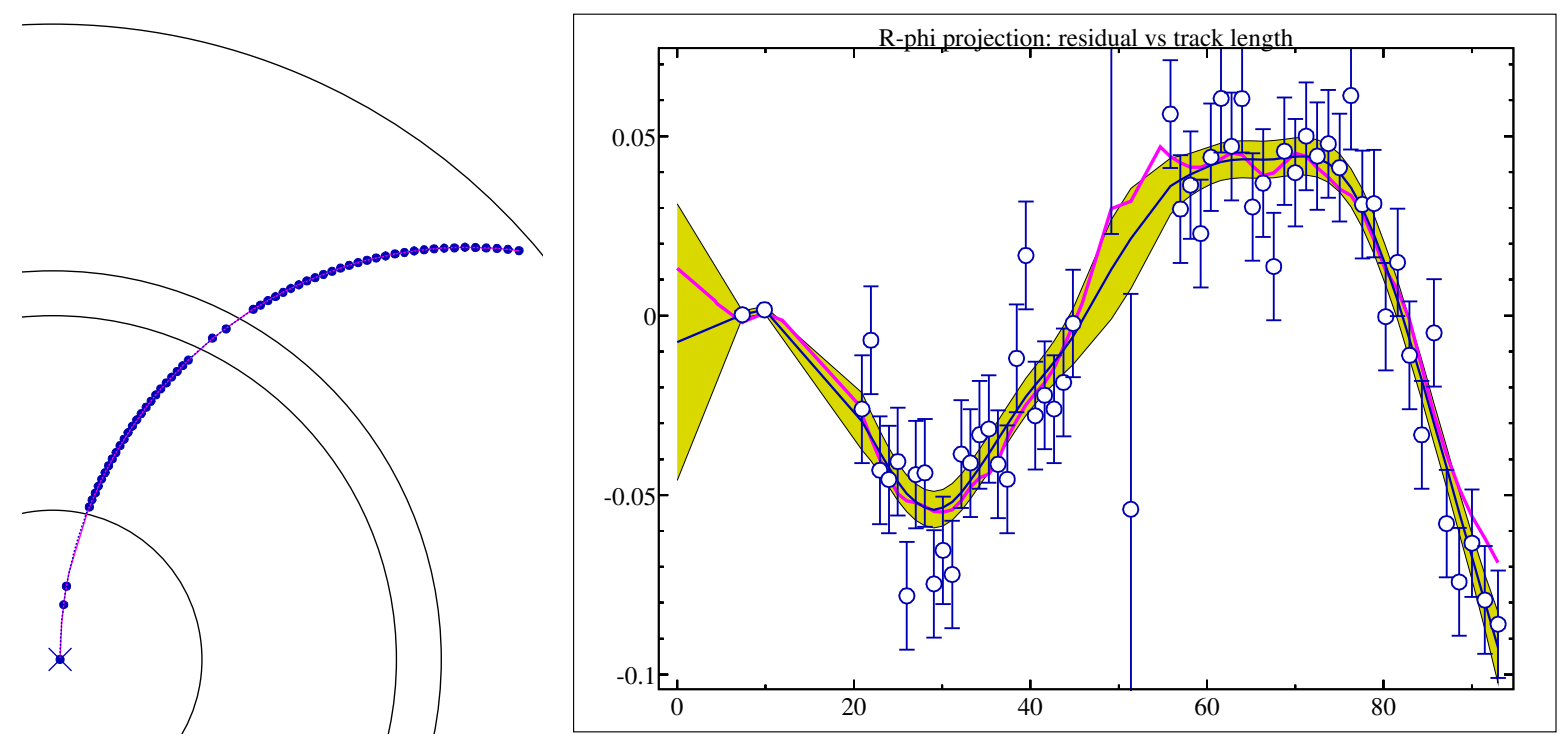

Fig. 4. A $200 \mathrm{MeV} / \mathrm{c}$ track in a detector, similar to the $\mathrm{H} 1$ detector. On the left the hits in the $r \phi$-plane (perpendicular to the magnetic-field direction) are show together with the result of a circle fit. The residuals of the hits w.r.t. the circle fit are shown on the right as a function of the track length. The lines and the band representing the result of the broken-line fit are explained in section 4.

$$
\boldsymbol{C}_{u}=\left(\begin{array}{ccccccc}
C_{11} & C_{12} & C_{13} & & & \\
C_{21} & C_{22} & C_{23} & C_{24} & & \\
C_{31} & C_{32} & C_{33} & C_{34} & C_{35} & \\
& C_{42} & C_{43} & C_{44} & C_{45} & \ldots \\
& & C_{53} & C_{54} & C_{55} & \ldots \\
& & & & & \ddots
\end{array}\right) .
$$

A fast solution with computing time $\propto n$ is based on the (Cholesky) decomposition of the matrix $\boldsymbol{C}_{u}$ according to $\boldsymbol{C}_{u}=\boldsymbol{L} \boldsymbol{D} \boldsymbol{L}^{\mathrm{T}}$, where the matrix $\boldsymbol{L}$ is a left unit triangular matrix (diagonal elements are 1) and $\boldsymbol{D}$ is a diagonal matrix; the band structure is kept in this decomposition, which can be made in-space, i.e. no extra storage is needed. The vector $\boldsymbol{u}$ is determined in the steps

$$
\begin{aligned}
& \text { decompose } & \boldsymbol{C}_{u} & =\boldsymbol{L} \boldsymbol{D} \boldsymbol{L}^{\mathrm{T}} \\
& \text { solve } & \boldsymbol{L} \boldsymbol{v} & =\boldsymbol{r}_{u} \\
& \text { solve } & \boldsymbol{L}^{\mathrm{T}} \boldsymbol{u} & =\boldsymbol{D}^{-1} \boldsymbol{v}
\end{aligned}
$$

The number of operations (multiplication, division) per step is indicated in the equations. In total $11 n$ operations are needed, to get the solution $\boldsymbol{u}$.

After the reconstruction of the trajectory the corrections $\Delta z_{0}$ and $\Delta(\tan \lambda)$ at track start are calculated from the first two $\boldsymbol{u}$-values $u_{1}$ and $u_{2}$ from the fitted trajectory and added to the initial approximations. In order to calculate the covariance matrix of the track parameters $z_{0}$ and $(\tan \lambda)$ only a few elements of the covariance matrix $\boldsymbol{V}_{u} \equiv$ $\boldsymbol{C}_{u}^{-1}$ are required. The method proposed by Takahashi et al. [6] can be used to calculate only those elements of the inverse matrix which are in the band of the original matrix, in a computation time linear in $n$, using the decomposition $\boldsymbol{L} \boldsymbol{D} \boldsymbol{L}^{\mathrm{T}}$. For the bandwidth of $m=2$ there are only $6 n$ operations to perform. The diagonal elements are the variances of the fitted values $u_{i}$.

\section{Tracking in the $r \phi$-plane}

Corrections to the parameters $\kappa, d_{\mathrm{ca}}$ and $\phi_{0}$ are determined in a fit to the residuals in the $r \phi$-plane; in addition to the parameters of section 3 there is a curvature correction $\Delta \kappa$. Corrections $\Delta d_{\text {ca }}$ and $\Delta \phi_{0}$ are calculated from the first two $\boldsymbol{u}$-values $u_{1}$ and $u_{2}$. The mean value of the kink angle $\beta_{i}$, as 
defined in Eq. (4), is now different from zero, due to the magnetic deflection. The magnetic deflection is taken into account by the re-definition of the kink angle

$$
\begin{array}{r}
\beta_{i}=\left[\begin{array}{l}
u_{i-1} \delta_{i-1}-u_{i}( \\
\left.\delta_{i-1}+\delta_{i}\right)+u_{i+1} \delta_{i}
\end{array}\right] \\
+\left(a_{i-1}+a_{i}\right) \cdot \Delta \kappa / 2
\end{array}
$$

(compare Eq. (4); $a_{i}$ is the distance between the points $i$ and $i+1)$ in the expression of Eq. (5), with $E\left[\beta_{i}\right]=0$. This has to be used in the function $S(\boldsymbol{u}, \Delta \kappa)$ to be minimized, which now depends on the additional parameter $\Delta \kappa$. The solution of the minimization problem is only slightly more complicated and time consuming, compared to the previous problem in section 3 . The linear least squares expression $S(\boldsymbol{u}, \Delta \kappa)$ is minimized by the solution of the matrix equation:

$$
\left(\begin{array}{c|c}
\boldsymbol{C}_{\kappa} & \boldsymbol{c}^{\mathrm{T}} \\
\hline \boldsymbol{c} & \boldsymbol{C}_{u}
\end{array}\right)\left(\begin{array}{c}
\frac{\Delta \kappa}{\boldsymbol{u}}
\end{array}\right)=\left(\begin{array}{l}
\frac{\boldsymbol{r}_{\kappa}}{\boldsymbol{r}_{u}} \\
\hline
\end{array}\right)
$$

where $\boldsymbol{C}_{u}$ is as before in section $3, \boldsymbol{C}_{\kappa}$ is a scalar and $\boldsymbol{c}$ is a vector. The solution with the steps

$$
\begin{aligned}
\boldsymbol{C}_{u} & =\boldsymbol{L} \boldsymbol{D} \boldsymbol{L}^{\mathrm{T}} \\
\boldsymbol{C}_{u} \boldsymbol{z} & =\boldsymbol{c} \\
\boldsymbol{B}_{\kappa} & =\left(\boldsymbol{C}_{\kappa}-\boldsymbol{c}^{\mathrm{T}} \boldsymbol{z}\right)^{-1} \\
\Delta \kappa & =\boldsymbol{B}_{\kappa}\left(\boldsymbol{r}_{\kappa}-\boldsymbol{z}^{\mathrm{T}} \boldsymbol{r}_{u}\right) \\
\boldsymbol{C}_{u} \widetilde{\boldsymbol{u}} & =\boldsymbol{r}_{u} \\
\boldsymbol{u} & =\widetilde{\boldsymbol{u}}-\boldsymbol{z} \Delta \kappa
\end{aligned}
$$

requires again a number of operations which is proportional to $n$. The variance of $\kappa$ is given by $\boldsymbol{B}_{\kappa}$. The submatrix $\boldsymbol{V}_{u}$ at the position of the matrix $\boldsymbol{C}_{u}$ in the inverse matrix is $\boldsymbol{C}_{u}^{-1}+\boldsymbol{z} \boldsymbol{B}_{\kappa} \boldsymbol{z}^{\mathrm{T}}$, which allows to calculate the covariance matrix of the parameters in a number of operations proportional to $n$. Parameter errors calculated by error propagation are in agreement with the width of pull distributions in a detector simulation. In Fig. 4 on the right the true simulated and the fitted trajectory are shown, together with the $\pm 1 \sigma$ band around the fitted trajectory.

\section{Summary}

The proposed algorithm allows fast track fits, fully taking into account multiple scattering; extensions to include energy loss and magnetic-field inhomogeneity are possible. The algorithm gives the full information on every measured point, the fitted value with propagated error, and the total $\chi^{2}(n-2$ degrees of freedom) with contributions from the two terms in the expressions $S(\boldsymbol{u})$ and $S(\boldsymbol{u}, \Delta \kappa)$. The deviation of each measured point can be checked individually. Pulls of position measurement and kink angle are easily calculated. Deviations of the pull distributions from the expected Gaussian shape with mean 0 and variance 1 would indicate deviations from the assumed measurement accuracy and the material assumption in the fit. Due to the efficient algorithms existing for band matrix operations the new algorithm is faster by a factor of six in comparison to the Kalman filter (under test conditions), and gives the result without iterations or recursion.

\section{References}

[1] Particle Data Group, Review of Particle Physics, Phys. Lett. B 592, 1 (2004)

[2] R. Mankel, Pattern recognition and event reconstruction in particle physics, Rep. Prog. Phys. 67, 553 (2004)

[3] P. Billoir, Track Fitting with Multiple Scattering: A New Method, Nucl. Instr. Meth. A 225, 352 (1984)

[4] R. Frühwirth, Application of Kalman filtering to track and vertex fitting, Nucl. Instr. Meth. A 262, 444 (1987)

[5] V. Karimäki, Effective circle fitting for particle trajectories, Nucl. Instr. Meth. A 305, 187 (1991)

[6] K. Takahashi, J. Fagan and M. Chin, Formation of a sparse bus impedance matrix and its applications to short circuit study, Proceedings 8th PICA Conference (1973), Minneapolis, Minnesota 